\title{
Randomised Double-Blind Trial of Combination Antibiotic Therapy in Rheumatoid Arthritis
}

\author{
Angela Smith, ${ }^{1}$ Caroline Doré, ${ }^{2}$ Peter Charles, ${ }^{1}$ Alena Vallance, ${ }^{3}$ Tara Potier, ${ }^{3}$ \\ and Charles Mackworth-Young ${ }^{1}$
}

${ }^{1}$ Kennedy Institute of Rheumatology, 1 Aspenlea Road, London W6 8LH, UK
${ }^{2}$ MRC Clinical Trials Unit, 222 Euston Road, London NW1 2DA, UK
${ }^{3}$ Charing Cross Hospital, Fulham Palace Road, London W6 8RF, UK

Correspondence should be addressed to Charles Mackworth-Young, c.mackworth-young@imperial.ac.uk

Received 30 March 2011; Accepted 4 May 2011

Academic Editor: Ronald F. van Vollenhoven

Copyright (c) 2011 Angela Smith et al. This is an open access article distributed under the Creative Commons Attribution License, which permits unrestricted use, distribution, and reproduction in any medium, provided the original work is properly cited.

\begin{abstract}
Objective. A combination of intravenous clindamycin and oral tetracycline has been used for many years as a treatment for active rheumatoid arthritis (RA), despite the absence of good evidence for its efficacy. A single-blind pilot study of this therapy suggested that a double-blind placebo-controlled trial was warranted. Methods. Patients with active RA were randomised in a $2: 1$ ratio to receive active treatment or placebo for 25 weeks. The active treatment consisted of intravenous clindamycin in a reducing regime, and oral tetracycline twice daily three times a week. 50 patients were to be recruited. The primary outcome measure was the proportion of patients achieving an ACR20 response. Results. An interim statistical analysis was performed after 20 patients had completed the study. Two patients in the active group achieved an ACR20 response, with none in the placebo group (NS). There was a better ESR20 response in the placebo group $(P=.02)$. There were no other significant differences between the groups. The results indicated that it was unlikely that a significant difference in ACR20 response would emerge if the remaining 30 patients were recruited. The trial was therefore halted. Conclusion. This antibiotic regime is unlikely to be a valuable therapy for active rheumatoid arthritis.
\end{abstract}

\section{Introduction}

Many drugs are used to treat RA. These include nonsteroidal anti-inflammatory drugs (NSAIDs), "second-line" drugs, corticosteroids and biological agents. All of these therapies have significant drawbacks in terms of efficacy, side effects, or cost $[1,2]$. For many patients; therefore, current therapy remains unsatisfactory, and there remains a need to find treatments that are effective, well tolerated, and relatively inexpensive.

There has been growing interest in the use of antibiotics for the treatment of RA, notably tetracyclines [3]. These were initially used by Brown et al. in uncontrolled studies in patients with RA [4]. The original rationale for their use was the hypothesis that RA may be caused by infection with Mycoplasma or similar organisms [5]. This now seems unlikely, and subsequent studies have suggested other actions for this class of drug, including the inhibition of matrix met- alloproteinases [6]. Three double-blind, placebo-controlled trials of minocycline in active RA have shown significant improvement in most clinical and laboratory measurements in the treatment group compared with the placebo group [7-9]. In the treatment of early RA, minocycline has also been shown to be superior to hydroxychloroquine [10] while doxycycline has been shown to enhance the response to methotrexate [11].

On the basis of other bacteria being a possible causative or exacerbating agent in RA [4], a combination of intravenous (IV) clindamycin and oral tetracycline has been used by some physicians for many years as a treatment for active rheumatoid arthritis. However, apart from unpublished, anecdotal reports, there was no controlled evidence for the efficacy of this regime. In view of this, we performed a singleblind, controlled pilot study of this antibiotic combination [12]. Half of the patients were given active treatment in addition to their usual therapy, and half were given no 
additional treatment. For ethical reasons patients in this latter group were not given a placebo, and all participants knew into which group they had been allocated. The treatment was given for a year, and patients were followed up for a further six months.

Nine out of eleven patients in the treatment group completed the first 12 months of the study, while only three of ten patients in the control group did $(P<.05)$. Patients who withdrew did so because of lack of improvement in their condition. Five patients in the treatment group had an ACR20 response at 12 months, while none of the control patients did $(P<.05)$. Improvement generally occurred within six months of starting the treatment. Further evidence for the efficacy of the treatment was that it was not well maintained once it had been withdrawn. No significant side effects were encountered.

These results suggested that this combined antibiotic therapy might be useful in the management of active RA although they could have been explained by a placebo response to the therapy. They indicated that a double-blind, placebo-controlled trial of the therapy was justified.

\section{Patients and Methods}

2.1. Patients. Male and female patients aged between 18 and 80 years attending the Rheumatology Clinic at Charing Cross Hospital were invited to take part in the study. All had classical RA of at least six months' duration, as defined by the American College of Rheumatology classification criteria [13]. All patients had active synovitis, a disease activity score (DAS28) of greater than 3.8 [14], and radiographic erosions. All patients had "failed" methotrexate or sulphasalazine: either they had had an inadequate response (as defined by persistence of active synovitis) to methotrexate (at least $7.5 \mathrm{mg}$ per week) or sulphasalazine (at least $1,000 \mathrm{mg}$ twice daily) for at least three months or they had been unable to take or tolerate either drug at the above doses. Patients could be taking other second-line antirheumatic drugs (e.g., sulphasalazine, methotrexate, hydroxychloroquine, azathioprine, leflunomide, intramuscular gold, oral gold, or penicillamine), but the dose of any of these had to have been unchanged for at least three months. They could also be on prednisolone, up to a maximum dose of $7.5 \mathrm{mg}$ per day, unchanged for at least three months. Patients could be taking NSAIDs and/or simple analgesics (e.g., paracetamol, coproxamol, or codeine).

Exclusion criteria included severely incapacity (Steinbrocker functional grade IV [15]), chronic/ recurrent infection (e.g., chronic bronchitis, recurrent sinusitis), other infections, immunodeficiency, malignancy, inflammatory bowel disease, other diarrhoeal states, other major illness, history of adverse reactions to tetracycline, clindamycin, or similar antibiotics, and pregnancy or lactation.

After recruitment, patients were randomly assigned in a 2: 1 ratio to one of two treatment groups: Group I (antibiotic group) and Group II (control group).

2.2. Treatment Regime: General. The patients in Group I received IV and oral antibiotics as below. Patients in Group
II received normal saline infusions and matched placebo tablets, as detailed below. Apart from the antibiotic therapy, the protocol for treatment (other drugs), assessment, and withdrawal from the study was identical for the two groups of patients. Patients were blinded as to which group they had been randomised, as were all clinicians and assessors.

2.3. Trial Drugs. The drug regime was the same as that commonly used in clinical practice (Hornett G, personal communication), and employed in the pilot study [12]. After an initial assessment (see below), patients in Group I started receiving IV infusions according to the regime in Table 1. Each infusion was given slowly in $250 \mathrm{~mL}$ normal saline over half an hour. Patients in Group II received IV saline without clindamycin according to the same regime. In addition, patients took oral tetracycline $250 \mathrm{mg}$ (Group I) or placebo (Group II) twice a day three times per week. This regime was continued for each patient for 25 weeks. There were a total of $18 \mathrm{IV}$ infusions for each patient. The maximum time between initial assessment and the first infusion was one month.

2.4. Other Drugs. During the study, the doses of secondline agents and prednisolone remained unaltered, if taken. If more active treatment of the rheumatoid arthritis was required, patients were offered joint aspiration and intraarticular injection of steroid (methylprednisolone 40-80 mg or hydrocortisone $12.5-25 \mathrm{mg}$ ) into one or more inflamed joints, up to a maximum of three injections. If further steroid was required or it became necessary to use another immunosuppressive drug, the patient left the study, and the event was recorded as a treatment failure. Patients were allowed to continue taking NSAIDs and/or simple analgesics and to alter the doses of these as required.

2.5. Withdrawal. Patients were free to withdraw from the study at any time and were withdrawn if this was thought necessary for any other reason by the supervising clinician.

2.6. Assessments. Patients were assessed at the outset of the study, and then every two months until the end of the study (25 weeks), that is, four assessments in all. The following were used:

2.6.1. Clinical. American College of Rheumatology (ACR) Core Data Set [16]; this included the following:

(i) patient's assessment of joint pain (visual analogue scale);

(ii) patient's global assessment of disease activity (visual analogue scale);

(iii) assessor's global assessment of disease activity (visual analogue scale);

(iv) functional assessment completion of health assessment questionnaire (HAQ) by patient; 
TABLE 1: Treatment and assessment regimes for patients in Group I (active treatment). Clindamycin infusions were given in $250 \mathrm{~mL}$ normal saline over one hour. Patients in Group II (placebo) received placebo infusions of normal saline and placebo tablets according to the same regime; they had the same assessment regime. bd: taken twice a day (morning and evening). $\times 3 /$ wk: three times per week. Assessments: $\mathrm{C}$, clinical; L, laboratory.

\begin{tabular}{|c|c|c|c|c|}
\hline \multirow{2}{*}{ Time } & \multirow{2}{*}{ Clindamycin infusion } & \multirow{2}{*}{ Tetracycline } & \multicolumn{2}{|c|}{ Assessment } \\
\hline & & & $\mathrm{C}$ & $\mathrm{L}$ \\
\hline Day 1 & $300 \mathrm{mg}$ & $250 \mathrm{mg}$ bd & + & + \\
\hline Day 2 & $300 \mathrm{mg}$ & none & & \\
\hline Day 3 & $600 \mathrm{mg}$ & $250 \mathrm{mg}$ bd & & \\
\hline Day 4 & $600 \mathrm{mg}$ & none & & \\
\hline Day 5 & $900 \mathrm{mg}$ & $250 \mathrm{mg}$ bd & & \\
\hline Weeks 2-4 & 900 mg per week & $250 \mathrm{mg}$ bd $\times 3 / \mathrm{wk}$ & & \\
\hline Weeks 5-8 & $900 \mathrm{mg}$ fortnightly & $250 \mathrm{mg}$ bd $\times 3 / \mathrm{wk}$ & & \\
\hline At week 9 & & & + & + \\
\hline Weeks 9-16 & $900 \mathrm{mg}$ fortnightly & $250 \mathrm{mg}$ bd $\times 3 / \mathrm{wk}$ & & \\
\hline At week 17 & & & + & + \\
\hline Weeks 17-24 & $900 \mathrm{mg}$ fortnightly & $250 \mathrm{mg}$ bd $\times 3 / \mathrm{wk}$ & & \\
\hline At week 25 & & & + & + \\
\hline
\end{tabular}

(v) number of swollen and number of tender joints (determined by physical examination of 28 joints).

NSAID and analgesic use.

The dose of oral prednisolone, and all other drugs. Any joints which had been injected with steroid during the study were excluded from the assessment.

2.6.2. Laboratory. Standard blood tests (full blood count, erythrocyte sedimentation rate (ESR), C-reactive protein (CRP), and liver function tests)

Serum matrix metalloproteinase levels (MMP-1 and MMP-3) were measured, using commercially available kits (R\&D Systems, Abingdon, UK). Urinary pyridinoline crosslinks (PYD) were detected using a specific monoclonal antibody in a commercial kit (Metra, Quidel, San Diego, Calif, USA) [17].

2.7. Outcome Measures. The primary outcome measure was the achievement of an ACR 20\% response [16] at six months. This was defined as:

(i) $>20 \%$ reduction in number of swollen and tender joints;

(ii) $>20 \%$ improvement in at least three out of five of the following:

(a) CRP;

(b) patient's assessment of joint pain (visual analogue scale);

(c) patient's global assessment of disease activity (visual analogue scale);

(d) assessor's global assessment of disease activity (visual analogue scale);

(e) functional assessment completion of health assessment questionnaire (HAQ) by the patient.
Other outcome measures included the following:

(i) individual components of the ACR score, as above;

(ii) withdrawal from the study because of a greater than 20\% deterioration according to ACR response;

(iii) number of intraarticular steroid injections given;

(iv) the use of NSAIDs and analgesics.

If withdrawal occurred for any reason, a final clinical and laboratory assessment was performed at this point.

\subsection{Statistics}

2.8.1. Randomization Ratio. A participant ratio of $2: 1$ between Groups I and II was chosen in order (i) to obtain more information on adverse effects in the treatment group, (ii) to aid recruitment, and (iii) to reduce withdrawals from the study.

2.8.2. Sample Size Calculations. The primary outcome measure was the proportion of patients who achieved an ACR $20 \%$ response. It was assumed that only $5 \%$ of the patients receiving standard therapy would respond. A sample size of 30 patients in Group I (active treatment) and 15 patients in Group II (placebo) would be sufficient to detect a response rate of $50 \%$ in the active-treated group using the 5\% significance level with a power of $80 \%$. The patient numbers were increased by approximately $10 \%$ to take account of individuals dropping out of the study for reasons other than disease progression. This gave 33 patients in Group I and 17 patients in Group II, and, therefore, a total of 50.

2.8.3. Statistical Analysis. The statistical analysis was restricted to the measurements at baseline and six months. For those patients who withdrew from the study, measurements from the final assessment were used in the statistical analysis. 
TABLE 2: Baseline characteristics of each group.

\begin{tabular}{lcccc}
\hline \multirow{2}{*}{ Variable } & \multicolumn{2}{c}{ Placebo $(n=8)$} & \multicolumn{2}{c}{ Active $(n=12)$} \\
& Mean & SD & Mean & SD \\
\hline Assess & 5.0 & 3.0 & 4.7 & 2.0 \\
DAS & 4.7 & 0.9 & 4.7 & 0.7 \\
EMS & 75 & 108 & 154 & 405 \\
HAQ & 1.7 & 0.4 & 1.3 & 0.7 \\
CRP & 12 & 15 & 23 & 19 \\
ESR & 29 & 16 & 26 & 16 \\
Fatigue & 6.6 & 1.6 & 6.3 & 1.5 \\
Pain & 4.2 & 2.7 & 5.2 & 1.9 \\
Pt. assess & 4.2 & 2.9 & 5.4 & 1.8 \\
Swollen joints & 11 & 4 & 12 & 5 \\
Tender joints & 6 & 3 & 7 & 3 \\
\hline
\end{tabular}

Assess: physician global assessment, VAS; DAS: DAS disease activity score; EMS: early morning stiffness; HAQ: HAQ disability score; Fatigue: fatigue assessment, VAS; Pain: patient assessment of pain, VAS; Pt. assess: patient global assessment, VAS; Swollen joints: swollen joint count; Tender joints: tender joint count.

The proportion of patients who achieved an ACR 20\% response (relative to baseline) at six months were compared in the two randomised groups, using Fisher's exact test. Patients who were withdrawn from the study were included in this analysis as nonresponders.

For continuous outcome measures a two-sample $t$-test was used to compare the change from baseline in the two randomised groups.

2.9. Ethical Approval. Ethical approval for this project was obtained from the Hammersmith Hospital Research Ethics Committee.

\section{Results}

It had been planned to recruit 50 patients to the trial: 33 patients in Group I (active treatment) and 17 patients in Group II (placebo). After the 20th patient had completed the study, an interim statistical analysis was performed. This analysis showed that in almost all outcome measures, there was no trend towards a difference between active treatment and placebo. The trial was, therefore, halted at this point. The following results are, therefore, those obtained on those 20 patients.

Twelve patients were randomised to active treatment and 8 to placebo treatment. Assessments were made at weeks 1, 8,16 , and 25 . Week 1 was the start of treatment and week 25 the end of treatment. Table 2 summarises the baseline characteristics of each group. The two randomised groups were similar at baseline.

The primary outcome measure was the ACR20 response at the end of treatment (week 25). Improvement was calculated by comparing the value at week 25 with the value at baseline (week 1). Improvements corresponded to a decrease for each of the 7 variables. These comparisons are summarised in Table 3. A total of 15 of the 20 patients completed the 25 weeks of followup. Patients who did not complete the treatment period were considered not to have achieved a $20 \%$ improvement. Two patients in the active group achieved an ACR20 response, while none of the patients in the placebo group did (not significant).

All five of the patients who did not complete the trial were on active therapy. Three patients were withdrawn due to disease progression, and two were withdrawn due to protocol transgression. This difference in completion rates was statistically significant $(P=.06)$. There was also a significant difference $(P=.02)$ in the percentage of patients with a $20 \%$ ESR response, with a higher improvement rate in the placebo group.

The sample size calculation assumed that $5 \%$ of placebo and $50 \%$ of active patients would achieve an ACR20 response, a difference in response rates of $45 \%$. The $95 \%$ confidence interval for the (placebo-active) difference in response rates was 38 to 4 , which excludes a difference of $45 \%$. The 95\% confidence interval for an ACR20 response in the active treatment group was 2 to $48 \%$, which excludes the response rate of 50\% used in the sample size calculation. This made it very unlikely that there would be a statistically significant difference in ACR20 response between the two groups if the trial were continued to completion and a total of 50 patients recruited.

An analysis of covariance was performed to compare the two treatments for the assessments at the end of trial, including the baseline value as a covariate. This enabled comparison of the final status of the two groups after allowing for their initial status. The results showed no discernable trend towards a difference between the two groups.

For the whole cohort of 20 patients, there was significant correlation between levels of MMP-1 and HAQ score ( $P=$ $.02)$, MMP- 3 and CRP $(P=.001)$, MMP-3 and patient global assessment $(P=.068)$, and PYD and HAQ $(P=.054)$. There was no significant correlation between MMP-1, MMP-3, and PYD levels, and any of the other measures of disease activity. There was no significant difference in the change in MMP-1, MMP-3, or PYD levels between the two groups.

\section{Discussion}

In this trial we used the same treatment regime-intravenous clindamycin and oral tetracycline-as that employed in the pilot study. Based on our power calculation, we had planned to recruit 50 patients in a $2: 1$ ratio (active to placebo treatment). However, after 20 patients had completed the study, an interim statistical analysis showed that it would be unlikely that a significant difference would emerge between the treatment groups if we were to recruit the remaining 30 patients. The trial was therefore halted.

The results reported here on the first 20 patients did show some differences between the two groups. Two out of the 12 patients (17\%) in the active treatment group (Group I) showed an ACR20 response, while none of the patients receiving placebo did (Group II). However, the confidence intervals were wide. If this trend were continued, we would have had to recruit 126 patients in order for the difference to become significant. 
TABLE 3: Number and percentage of patients with an ACR20 response, together with the number of patients with a $20 \%$ improvement for each core variable. 20 after each variable indicates $20 \%$ improvement.

\begin{tabular}{|c|c|c|c|c|c|c|c|}
\hline \multirow{2}{*}{ Variable } & \multicolumn{2}{|c|}{ Placebo $(n=8)$} & \multicolumn{2}{|c|}{ Active $(n=12)$} & \multicolumn{2}{|c|}{ Placebo-active } & \multirow[t]{2}{*}{$P$ value } \\
\hline & Number & $\%$ & Number & $\%$ & Difference \% & $95 \% \mathrm{CI}$ & \\
\hline ACR20 & 0 & 0 & 2 & 17 & -17 & -38 to 4 & .5 \\
\hline Completed & 8 & 100 & 7 & 58 & 42 & 14 to 70 & .06 \\
\hline HAQ20 & 0 & 0 & 2 & 17 & -17 & -38 to 4 & .5 \\
\hline VAS Assess.20 & 5 & 63 & 4 & 33 & 29 & -14 to 72 & .4 \\
\hline Pat. Pain 20 & 1 & 13 & 3 & 25 & -13 & -46 to 21 & .6 \\
\hline Pat. Dis. Assess. 20 & 1 & 13 & 2 & 17 & -4 & -35 to 27 & 1.0 \\
\hline CRP20 & 3 & 38 & 4 & 33 & 4 & -39 to 47 & 1.0 \\
\hline ESR20 & 5 & 63 & 1 & 8 & 54 & 17 to 91 & .02 \\
\hline Swollen joint 20 & 4 & 50 & 6 & 50 & 0 & -45 to 45 & 1.0 \\
\hline Tender joint 20 & 6 & 75 & 6 & 50 & 25 & -16 to 66 & .4 \\
\hline
\end{tabular}

Against this trend, the results showed a higher number of withdrawals due to disease progression in Group I compared with Group II. There was a statistically significant greater improvement in ESR in Group II compared with Group I.

There are a number of possibilities to explain the apparent lack of benefit of the active treatment. It is likely that receiving intravenous infusions on a regular basis has a significant placebo effect. This could explain why there was an apparent benefit in the single-blind but not in the doubleblind study. It may be that a small number of patients with RA do genuinely respond to the antibiotic therapy, but that our study was underpowered to detect this. If this is the case, it could be argued that the number needed to treat would be too high for this to be a valuable therapy. Another possibility is the difference in oral antibiotic: previous studies in this field have generally used minocycline or doxycycline [9-13], and the dose of tetracycline used in this trial was comparatively low; in earlier studies, minocycline and doxycycline have been given twice daily [9-13].

The literature shows robust evidence for the efficacy of various tetracycline-based antibiotic regimes in modifying the activity of rheumatoid arthritis. The results of the present trial would be consistent with the hypothesis that it is the anti-inflammatory rather than the antibacterial activity of tetracyclines that confers this benefit.

The particular combination of clindamycin and tetracycline used in this trial has been employed extensively in clinical practice. However, the results indicate that it may not be suitable for further study.

\section{Acknowledgment}

This study was kindly supported by a grant from the Peacock Trust, 4 Waterstone Close, Itchenor, West Sussex P. O. Box 20 7BP, UK

\section{References}

[1] N. J. Olsen and C. M. Stein, "New drugs for rheumatoid arthritis," New England Journal of Medicine, vol. 350, no. 21, pp. 2167-2179, 2004.
[2] J. R. O'Dell, “Therapeutic strategies for rheumatoid arthritis," New England Journal of Medicine, vol. 350, no. 25, pp. 25912602, 2004.

[3] M. Stone, P. R. Fortin, C. Pacheco-Tena, and R. D. Inman, "Should tetracycline treatment be used more extensively for rheumatoid arthritis? Meta-analysis demonstrates clinical benefit with reduction in disease activity," Journal of Rheumatology, vol. 30, no. 10, pp. 2112-2122, 2003.

[4] TM Brown, SW Bush, and WR Felts, "Management of the chronically ill patient," in Long Term Illness, M. G. Wohe, Ed., WB Saunders, Philadelphia, Pa, USA, 1959.

[5] D. K. Ford, "The microbiological causes of rheumatoid arthritis," Journal of Rheumatology, vol. 18, no. 10, pp. 14411442, 1991.

[6] A. N. Sapadin and R. Fleischmajer, "Tetracyclines: nonantibiotic properties and their clinical implications," Journal of the American Academy of Dermatology, vol. 54, no. 2, pp. 258-265, 2006.

[7] M. Kloppenburg, F. C. Breedveld, J. P. Terwiel, C. Mallée, and B. A. C. Dijkmans, "Minocycline in active rheumatoid arthritis," Arthritis and Rheumatism, vol. 37, no. 5, pp. 629636, 1994.

[8] B. C. Tilley, G. S. Alarcon, S. P. Heyse et al., "Minocycline in rheumatoid arthritis. A 48-week, double-blind, placebocontrolled trial," Annals of Internal Medicine, vol. 122, no. 2, pp. 81-89, 1995.

[9] J. R. O’Dell, K. M. Blakely, W. Palmer et al., “Treatment of early rheumatoid arthritis with minocycline or placebo: results of a randomized, double-blind, placebo-controlled trial," Arthritis and Rheumatism, vol. 40, no. 5, pp. 842-848, 1997.

[10] J. R. O’Dell, K. M. Blakely, J. A. Mallek, P. J. Eckhoff, R. D. Leff et al., "Treatment of early seropositive rheumatoid arthritis: a two-year, double-blind comparison of minocycline and hydroxychloroquine," Arthritis and Rheumatism, vol. 44, no. 10, pp. 2235-2241, 2001.

[11] J. R. O’Dell, J. Elliott, J. A. Mallek et al., “Treatment of early seropositive rheumatoid arthritis: doxycycline plus methotrexate versus methotrexate alone," Arthritis and Rheumatism, vol. 54, no. 2, pp. 621-627, 2006.

[12] L. L. Gompels, A. Smith, W. Rogers et al., "Single-blind randomized trial of combination antibiotic therapy in rheumatoid arthritis," Journal of Rheumatology, vol. 33, no. 2, pp. 224227, 2006. 
[13] F. C. Arnett, S. M. Edworthy, D. A. Bloch et al., "The American Rheumatism Association 1987 revised criteria for the classification of rheumatoid arthritis," Arthritis and Rheumatism, vol. 31, no. 3, pp. 315-324, 1988.

[14] M. Prevoo, M. A. van 't Hof, H. H. Kuper, M. A. Van Leeuwen, L. B. A. Van De Putte, and P. L. C. M. Van Riel, "Modified disease activity scores that include twenty-eight-joint counts," Arthritis and Rheumatism, vol. 38, no. 1, pp. 44-48, 1995.

[15] O. Steinbrocker, C. H. Traeger, and R. Batterman, "Therapeutic criteria in rheumatoid arthritis," Journal of the American Medical Association, vol. 140, no. 8, pp. 659-662, 1949.

[16] D. T. Felson, J. J. Anderson, M. Boers et al., "American College of Rheumatology preliminary definition of improvement in rheumatoid arthritis," Arthritis and Rheumatism, vol. 38, no. 6, pp. 727-735, 1995.

[17] G. Kallerup, M. Hansuren, and K. Horsleu-Peterson, "Urinary hydroxypyridinium cross-links of collagen in rheumatoid arthritis: relation to disease activity and effects of methyl prednisolone," British Journal of Rheumatology, vol. 33, no. 9, pp. 816-820, 1994. 


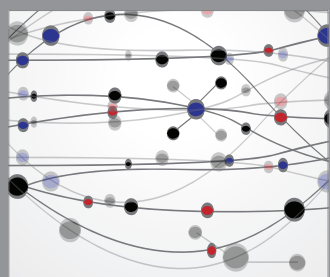

The Scientific World Journal
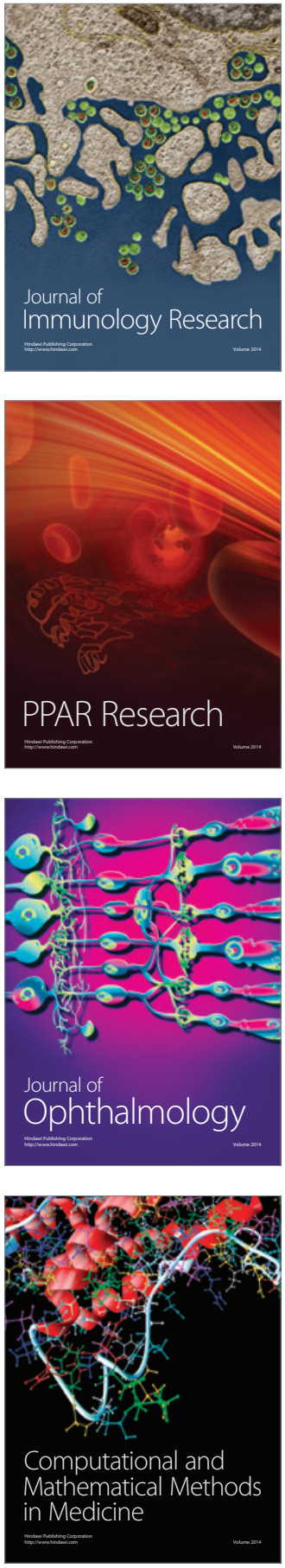

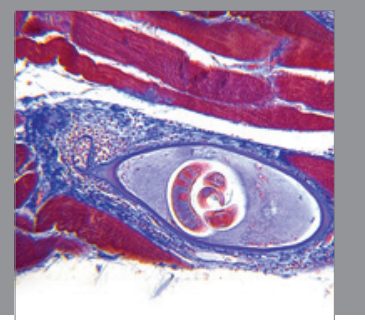

Gastroenterology

Research and Practice
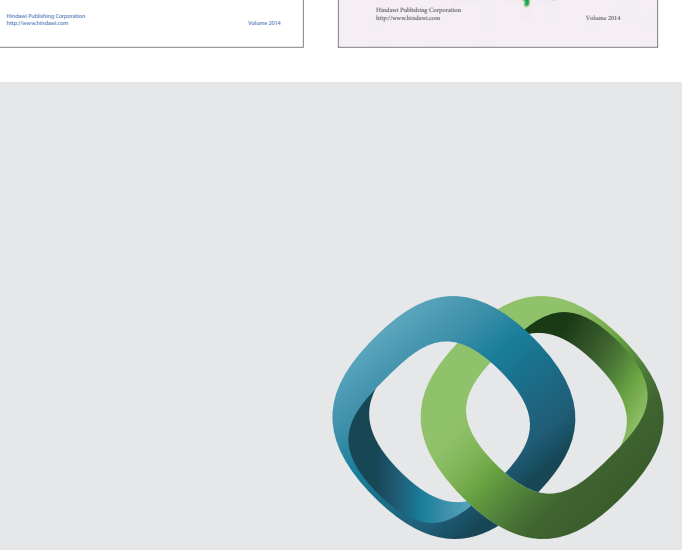

\section{Hindawi}

Submit your manuscripts at

http://www.hindawi.com
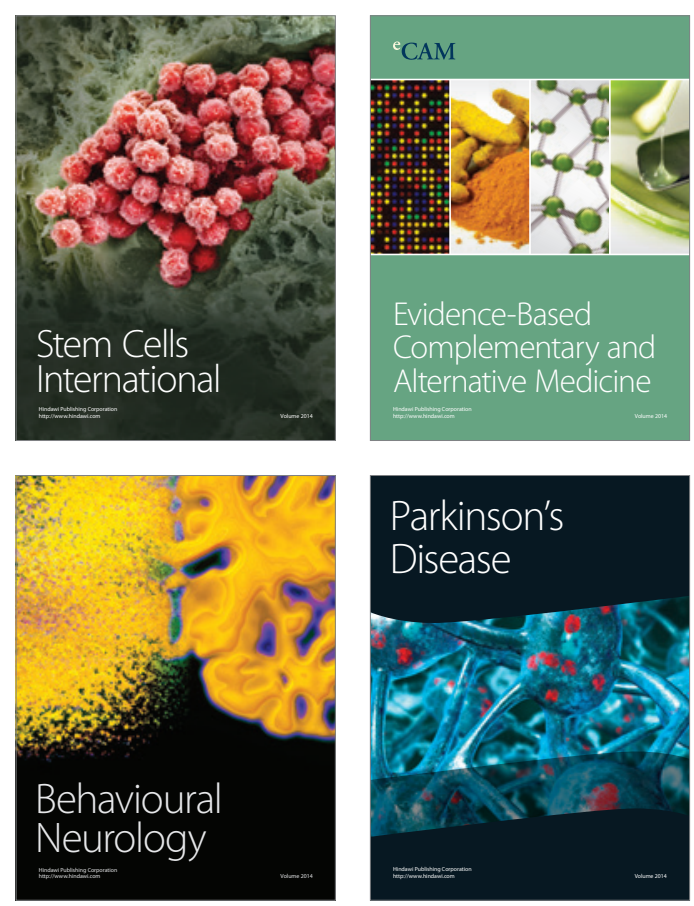

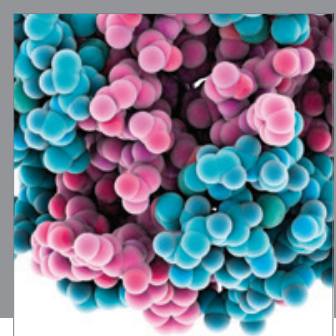

Journal of
Diabetes Research

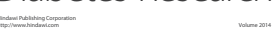

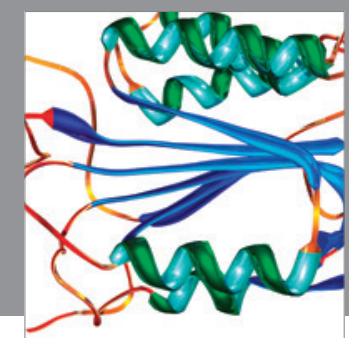

Disease Markers
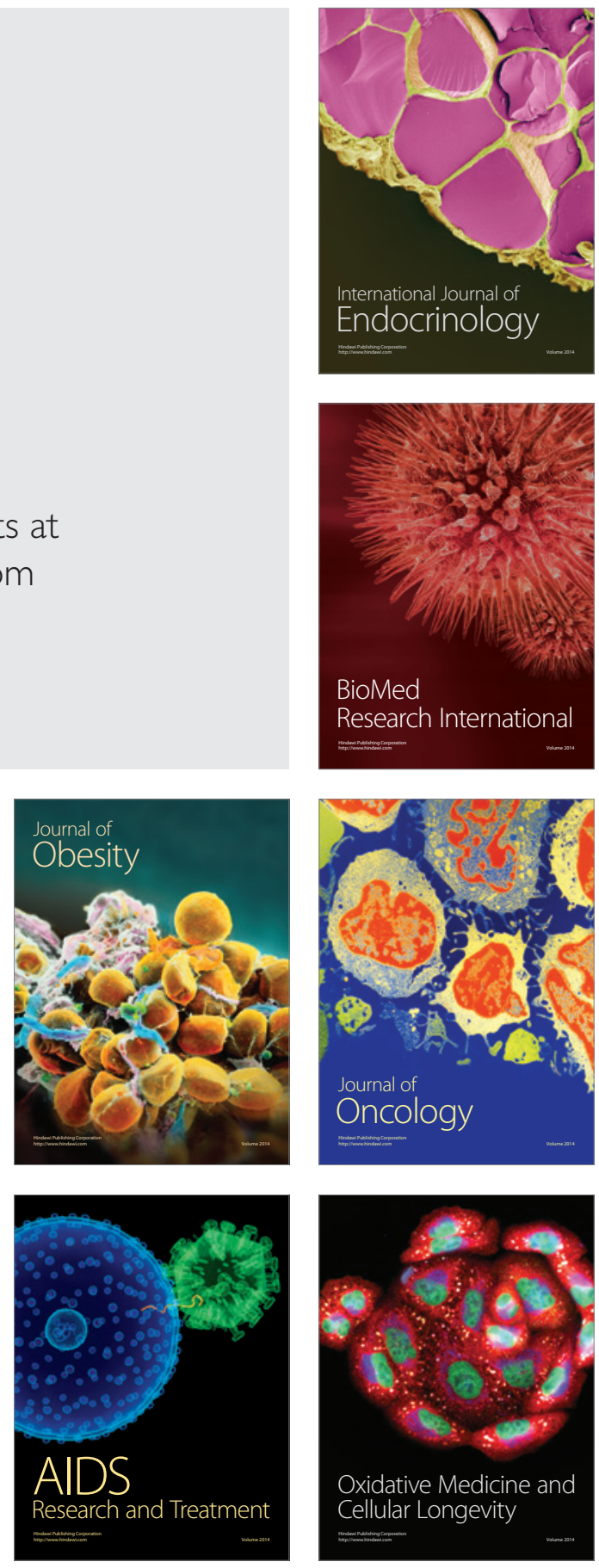